\title{
Morphological and functional changes associated with a 12-week self-guided exercise programme of overweight adults: a pilot study
}

\author{
A. D. Stewart ${ }^{1}$, C. Rolland ${ }^{1}$, A. Gryka ${ }^{1}$, S. Smith ${ }^{2}$, J. Jones ${ }^{2}$, S. Findlay ${ }^{2}$ and HIM. Davidson ${ }^{2}$ \\ ${ }^{1}$ Centre for Obesity Research and Epidemiology, Robert Gordon University, St Andrew Street, Aberdeen AB25 $1 H G$, UK \\ and ${ }^{2}$ Dietetics, Nutrition \& Biological Sciences, Queen Margaret University, Musselburgh EH21 $6 U U$
}

Walking-based exercise interventions in a community setting have failed to show improvement in health parameters over 12 weeks ${ }^{(1)}$. Conversely, carefully controlled exercise of sufficient intensity and duration in overweight and obese adults has been shown to induce substantial weight and fat losses within the same time interval ${ }^{(2)}$. There is likely to be a threshold of exercise stimulus, above which functional changes and health benefits can typically be anticipated. However, it is unknown if sedentary overweight individuals who undertake self-managed structured exercise over 12 weeks will reach this threshold. Therefore, the aim of this study was to determine if changes occur in morphological and health parameters within this time frame in response to the introduction of self-guided exercise.

Twenty three volunteers between 40 and $60 \mathrm{y}$ who passed all aspects of the physical activity readiness questionnaire (PARQ) but were not meeting current UK physical activity recommendations, were recruited from two centres. Those on medication for heart disease; diabetes; hypertension; already engaging in structured exercise or walking $>2$ miles per week were excluded. Before and after the intervention, each was assessed for stature, mass, waist, sagittal abdominal diameter (SAD), triceps and medial calf skinfolds (and abdominal skinfold in centre 1), sit-to-stand $60 \mathrm{~s}$ test, blood pressure, and completed questionnaires on quality of life and fatigue.

Across both centres, 3 male and 14 female participants (aged 47.5 (SD 5.1) y completed the study. At baseline, body mass was 89.8 (SD 21.3) kg, BMI was 33.2 (SD 6.8) kg.m ${ }^{-2}$, waist was 99.5 (SD 14.6) cm; SAD was 30.5 (SD 5.8) cm. At 12 weeks, mass had reduced by 1.8 (SD 1.7) kg $(P<0.0001)$; BMI reduced by 0.7 (SD 0.6$) \mathrm{kg} . \mathrm{m}^{-2}$; waist reduced by 4.1 (SD 3.8$) \mathrm{cm}(P<0.0001)$ and SAD reduced by 0.9 (SD 1.0) $\mathrm{cm}(P=0.002)$. Reduction in skinfolds was not significant. Mean $\%$ changes at 12 weeks were $2.1,3.1$ and $4.0 \%$ for mass, SAD and waist respectively. These morphological changes appear modest but significant. The fact that both waist and SAD changes were significant in the absence of commensurate adjustments in skinfolds suggests that visceral fat may have reduced as a consequence of this intervention. This is consistent with evidence that exercise preferentially mobilises visceral fat. It remains to be determined if alterations in diet may have contributed to the changes observed. Because clear health improvements may be unaccompanied by significant subcutaneous adipose tissue reduction, these findings have important implications for those pursuing self-guided exercise. The temporal changes in visceral and subcutaneous fat, alongside the fatigue and quality of life remain important considerations for future research.

1. Baker G, Gray SR, Wright A et al. for the Scottish Physical Activity Research Collaboration. (2008). Int J Behavioral Nutr and Physical Activity 5, 44 doi:10.1186/1479-5868-5-44.

2. King NA, Hopkins M, Caudwell P et al. (2008) Int J Obes 32, 177-184. 